\title{
DESIGN OF AN ALL-OPTICAL WDM LIGHTPATH CONCENTRATOR
}

\author{
Shivashis Saha ${ }^{\dagger}$, Eric D. Manley ${ }^{\star}$, Jitender S. Deogun ${ }^{\dagger}$ \\ ${ }^{\dagger}$ Department of Computer Science and Engineering, University of Nebraska-Lincoln, Lincoln, NE 68588-0115, U.S.A. \\ ${ }^{\star}$ Department of Mathematics and Computer Science, Drake University, Des Moines, IA 50311, U.S.A. \\ Email: ssaha@cse.unl.edu, eric.manley@drake.edu,deogun@cse.unl.edu
}

\begin{abstract}
A design of a nonblocking, all-optical lightpath concentrator using wavelength exchanging optical crossbars and and WDM crossbar switches is presented. The proposed concentrator is highly scalable, cost-efficient, and can switch signals in both space and wavelength domains without requiring a separate wavelength conversion stage.
\end{abstract}

\section{KEY WORDS}

Optical Networks, Lightpath Concentrator, Network Interfaces, Non-blocking

\section{Introduction}

A concentrator is a switch in which the number of input fibers is strictly greater than the number of output fibers. Thus, a concentrator drops some input signals and switches each of the remaining signals to output fibers on possibly different wavelengths. If the switching is done in the electronic domain, it is called an electronic concentrator or simply a concentrator. If the switching is performed in all-optical domain, then it is called an all-optical lightpath concentrator or simply lightpath concentrator.

With the advancement of wavelength division multiplexing (WDM) technology, there has been an exponential growth in the size of interconnects, their complexity, the number of necessary optical-electrical-optical $(\mathrm{O} / \mathrm{E} / \mathrm{O})$ converters, and their cost. There is a large mismatch between the capacity and speed of optical transmission compared with electronic transmission which results in a bottleneck of the electronic switches and concentrators in WDM networks. This mismatch motivates design of all-optical switches and lightpath concentrators which can keep data in the optical domain, eliminating the need for costly and inefficient $\mathrm{O} / \mathrm{E} / \mathrm{O}$ conversions.

There are several practical deployments of optical networks where it is necessary to switch signals from $N$ fibers to $M$ fibers, where $N>M$ [1]. For example, the function of a hub in metro networks is to collect $N$ lightpaths from several regional hubs and transfer them to $M$ lightpaths in the backbone network. Thus, a hub is a lightpath concentrator [1]. Lightpath concentrators have also been used in the design of all-optical switch architectures [2], [3], [4].

With the advancement of WDM technologies, there has been a need for cost effective WDM interconnects. WDM crossconnects typically have separate stages for space and wavelength switching. These designs are expensive, and the switching costs increase significantly with the increase in the number of lightpaths carried by the fiber. A new paradigm for WDM interconnect design is evolving in which space and wavelength switching is performed simultaneously and seamlessly [2], [3]. This design for WDM crossconnects eliminates the need for separate wavelength conversion stages.

In this paper, we present a design of an all-optical lightpath concentrator based on wavelength exchanging optical crossbars (WOC) and WDM Crossbar Switches [2], [3]. The design of an all-optical lightpath concentrator presented in this paper makes use of WOC properties and can similarly achieve a balance between wavelength switching and conversion costs and does not require a separate wavelength conversion stage.

\section{Related Work}

A design of a WDM Optical Interconnect based on the concept of concentrators was proposed in [4]. This switch achieves full connectivity by using low cost limited wavelength converters. However, the design is based on the traditional concept of performing space and wavelength switching in separate stages [5]. A new class of WDM crossconnects using WOCs that minimizes the switching and wavelength conversion costs was proposed in [2].

Three designs of all-optical lightpath concentrators were presented in [1]. The first design is a generalization of the optical crossconnects which requires $M \times M$ optical switches and has the smallest blocking probability. The second, design based on the concept of partial concentrators, is less costly but has larger blocking probability. In the third design, small lightpath concentrators are used as building blocks to construct a large concentration network. Since the building blocks are blocking, the third design is also blocking. Moreover these designs perform only space switching and do not consider wavelength switching at all.

\subsection{Preliminaries}

A lightpath concentrator with $F_{1}$ incoming fibers and $F_{2}$ outgoing fibers, where each fiber carries $W$ wavelengths is called an $N \times M$ lightpath concentrator, where $N=F_{1} W$, $M=F_{2} W$, and $N>M$. For the sake of simplicity we assume $F_{1}, F_{2}$, and $W$ are all powers of 2 . The set of 
incoming fibers is given as $F_{1}=\left\{f_{11}, f_{12} \ldots f_{1 F_{1}}\right\}$, the set of outgoing fibers as $F_{2}=\left\{f_{21}, f_{22} \ldots f_{2 F_{2}}\right\}$, and the set of wavelengths as $W=\left\{\lambda_{1}, \lambda_{2} \ldots \lambda_{W}\right\}$.

If an $N \times M$ lightpath concentrator can achieve any permutations of at most $M$ out of $N$ input lightpaths, it is called nonblocking, otherwise it is called blocking.

A wavelength request model $\left(\lambda_{i}, f_{1 x}, \lambda_{j}, f_{2 y}\right)$, $\lambda_{i}, \lambda_{j} \in W, f_{1 x} \in F_{1}$, and $f_{2 y} \in F_{2}$, means an incoming lightpath on fiber $f_{1 x}$ at wavelength $\lambda_{i}$ is to be routed to an output fiber $f_{2 y}$ at wavelength $\lambda_{j}$ [2].

A new class of WDM crossconnects denoted by $W^{\lambda}(F \times F)$ was proposed in [2]. This crossconnect has $F$ input and $F$ output fibers, each carrying $W$ wavelengths $(N=F W)$. The design of this class is based on the basic $2 \times 2$ switch called WOC [2]. A WOC acts like bar state of a traditional crossbar when the control signal is off (see Figure 1(a)). Otherwise, it performs both space switching and wavelength conversion simultaneously (see Figure 1(b)). The design of a $2^{\lambda}(2 \times 2)$ (i.e. $W=2, F=$ $2, N=2 \times 2=4)$ WDM crossbar switch is shown in Figure 2 . The rectangles are switching elements and circles denote WOCs.

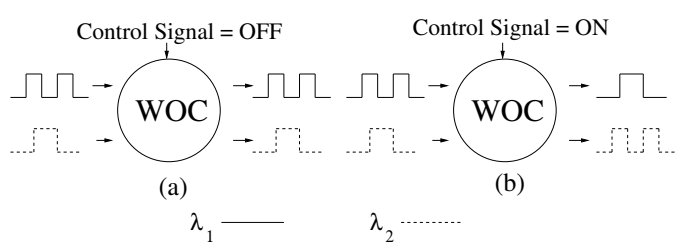

Figure 1. (a) WOC in bar state (b) WOC in cross state [2]
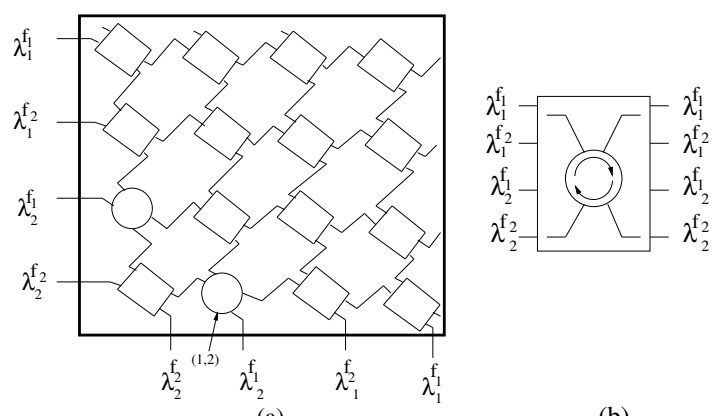

(a)

(b)

Figure 2. (a) $2^{\lambda}(2 \times 2) \mathrm{WDM}$ crossbar switch. The rectangles represent $2 \times 2$ space switches while the circles represent WOCs. (b) Symbolic notation for the $2^{\lambda}(2 \times 2)$ WDM crossbar switch. [2]

The concept of WOC is based on the simultaneous exchange of power between two signals which has been experimentally demonstrated using four wave mixing $(F W M)$, photonic crystals and nano-photonic devices [2], [6]. The conversion and switching speeds of WOC are determined by the modulation speed of its pump [2]. Crosstalk may be a problem in WOCs because of the power exchanging process, but with the improvement and advances in the photonic and nano technologies, efficient design of WOC is possible in the near future.

\section{Proposed Design}

In this section, we discuss the design of the proposed alloptical lightpath concentrator and its scalability issues.

\subsection{Basic Lightpath Concentrator}

A basic $2^{\lambda}\left(N \times \frac{N}{2}\right)$ lightpath concentrator is a device which can switch lightpaths from 2 wavelengths on $N$ input fibers (where $N$ is a power of 2) to 2 wavelengths on $\frac{N}{2}$ output fibers. Basic lightpath concentrators can be used as the building blocks for designs of larger lightpath concentrators. The design of a basic $2^{\lambda}(2 \times 1)$ lightpath concentrator is shown in Figure 3. This is the smallest lightpath concentrator used in the recursive design of all larger concentrators. For $N>2$, a basic $2^{\lambda}\left(N \times \frac{N}{2}\right)$ concentrator can be constructed recursively by using two $1^{\lambda}(N \times N)$ WDM crossbar switches and two basic $2^{\lambda}\left(\frac{N}{2} \times \frac{N}{4}\right)$ lightpath concentrators. The design of the basic $2^{\lambda}(4 \times 2)$ concentrator is shown in Fig. 4.

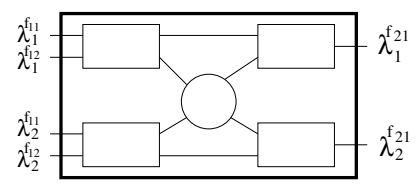

(a)

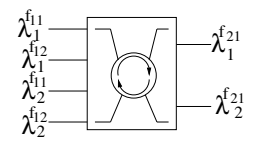

(b)

Figure 3. (a) The basic $2^{\lambda}(2 \times 1)$ lightpath concentrator (b) Its symbolic notation

\subsection{Scalability of the Concentrator Design}

Now that we have presented the design of a basic $2^{\lambda}(N \times$ $\frac{N}{2}$ ) lightpath concentrator, we will show how this can be scaled to a $W^{\lambda}\left(N \times \frac{N}{2}\right)$ concentrator to support an arbitrary number of wavelengths. We then describe how this can be extended to $W^{\lambda}(N \times M)$ concentrators.

\subsection{1 $W^{\lambda}\left(N \times \frac{N}{2}\right)$ Lightpath Concentrator}

The $W^{\lambda}\left(N \times \frac{N}{2}\right)$ again uses a recursive 2-stage design. The first stage uses WDM crossbar switches, and the second 


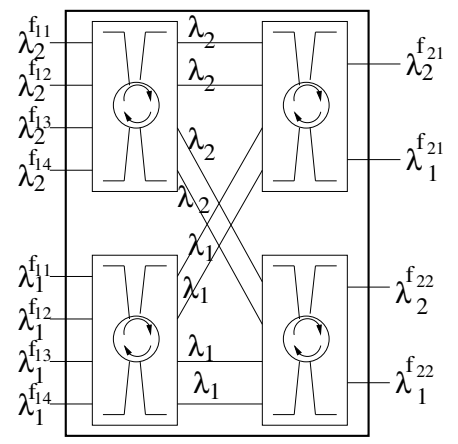

Figure 4 . The basic $2^{\lambda}(4 \times 2)$ lightpath concentrator

stage uses smaller lightpath concentrators which operate on fewer wavelengths. The first stage makes sure that the signal is properly switched in space, and wavelength is converted to an appropriate wavelength depending on the output port (although this may not be the actual output wavelength). The second stage guarantees the input lightpath is properly wavelength switched and routed to the desired output port. This design requires two $\left(\frac{W}{2}\right)^{\lambda}(N \times N)$ WDM crossbar switches, and two $\left(\frac{W}{2}\right)^{\lambda}(N \times M)$ lightpath concentrators. Applied recursively (when $W$ is a power of 2), this will eventually require the use of $2^{\lambda}(N \times M)$ concentrators which were presented in the previous section. The design of the $4^{\lambda}(4 \times 2)$ lightpath concentrato is shown in Figure 5.

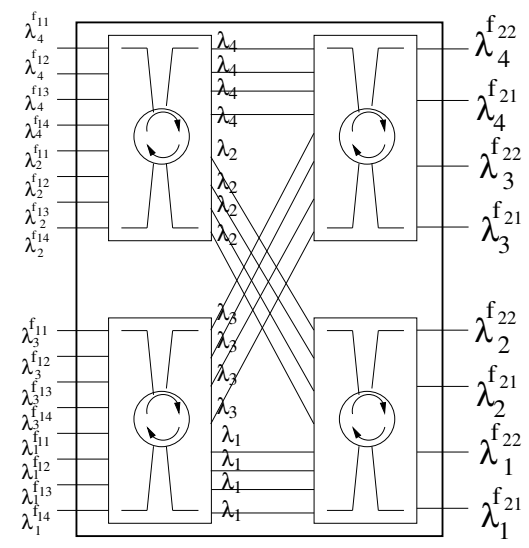

Figure 5. $4^{\lambda}(4 \times 2)$ lightpath concentrator

\subsection{2 $W^{\lambda}(N \times M)$ Lightpath Concentrator}

Again, we assume that $W, N$, and $M$ are powers of 2, but $N \neq 2 M$. Let $N=2^{n}$ and $M=2^{m}$. Then a $W^{\lambda}(N \times M)$ can be immediately constructed in stages with one $W^{\lambda}\left(2^{n} \times 2^{m+1}\right)$ concentrator followed by one $W^{\lambda}\left(2^{m+1} \times 2^{m}\right)$ concentrator. However, the WDM crossbars can be eliminated from each of these two subconcentrators if we instead replace the first stage with two $\left(\frac{W}{2}\right)^{\lambda}\left(2^{n} \times 2^{m+1}\right)$ concentrators and the second stage by two $\left(\frac{W}{2}\right)^{\lambda}\left(2^{m+1} \times 2^{m}\right)$ concentrators. For example, see the $4^{\lambda}(4 \times 1)$ lightpath concentrator in Fig. 6 .

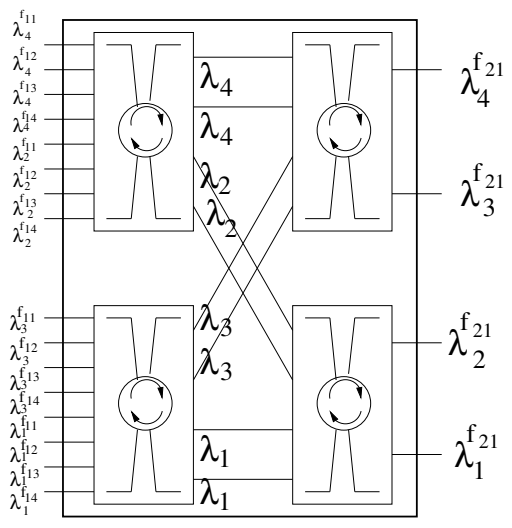

Figure 6. $4^{\lambda}(4 \times 1)$ lightpath concentrator

\section{Properties of the Proposed Design}

In this section, we establish two important properties of our all-optical lightpath concentrator design.

Proposition 1: The proposed design of a basic $2^{\lambda}\left(N \times \frac{N}{2}\right)$ lightpath concentrator is nonblocking in both space and wavelength domains.

It is easy to see by inspection that any combination of the four input signals can be received on the two outputs of the $2^{\lambda}(2 \times 1)$ concentrator. Arguing inductively, we assume that the smaller $2^{\lambda}\left(\frac{N}{2} \times \frac{N}{4}\right)$ concentrators used in the design are nonblocking in space and wavelengths. Furthermore, the two switches in the first stage are nonblocking space switches. Each of the two second-stage concentrators can then receive as inputs any combination of the $\frac{N}{2}$ inputs from the each of the first stage switches. From among these, because the smaller concentrators are nonblocking, any $\frac{N}{4}$ of these can be output on any desired wavelength/fiber combination. Thus, any combination of $\frac{N}{2}$ of the inputs to the $2^{\lambda}\left(N \times \frac{N}{2}\right)$ concentrator my be output in any permutation of the outputs.

Proposition 2: The proposed design of an all-optical $W^{\lambda}(N \times M)$ lightpath concentrator is nonblocking in both space and wavelength domains.

The proof is essentially the same as the proof for Proposition 1. We assume that each of the four switches and smaller concentrators in the design are nonblocking. Since each concentrator in the second stage can receive any $M / 2$ outputs from each first-stage component, by the inductive hypothesis, any combination of $M$ inputs can be output on any permutation of the outputs.

From the recursive nature of the construction of the lightpath concentrator it follows that if a signal has to be dropped, it can be dropped by the lightpath concentrator at the last possible stage. An algorithm can be easily implemented to drop signals by the lightpath concentrator based 
on a given criteria. We briefly present a routing algorithm for the proposed design in Figure 7.

Routing Algorithm for a request $\left(\lambda_{i}, f_{1 x}, \lambda_{j}, f_{2 y}\right)$

1. If all connections from the first-stage component connected to input $\left(\lambda_{i}, f_{1 x}\right)$ to the second stage component connected to output $\left(\lambda_{j}, f_{1 y}\right)$ are unavailable, then run the algorithm recursively for the first-stage component on request $\left(\lambda_{i}, f_{1 x}, \lambda_{k}, f_{2 z}\right)$ for each $\left(\lambda_{k}, f_{2 z}\right)$ unavailable output port of the first-stage component. Use the route which drops the signal at the last possible stage among these options.

2. Otherwise, route the signal along some available connection which connects the first-stage component connected to input $\left(\lambda_{i}, f_{1 x}\right)$ to the secondstage component connected to output $\left(\lambda_{j}, f_{1 y}\right)$. Suppose this input port on the second-stage component is $\left(\lambda_{l}, f_{1 w}\right)$. Then, call the routing algorithm recursively on the second-stage component for request $\left(\lambda_{l}, f_{1 w}, \lambda_{j}, f_{2 y}\right)$.

Figure 7. Routing algorithm for an all-optical $N \times M$ lightpath concentrator

\section{Conclusion and Future Work}

With the advancement of WDM technologies, there has been an emerging need for efficient and cost effective design of an all-optical lightpath concentrator. The increasing mismatch between the speed of optical and electronic transmission and the expensive O/E/O conversion cost has motivated our interest in the design of an all-optical lightpath concentrator, where the lightpath always remains in the optical domain. WDM interconnects based on traditional switching requires separate space and wavelength switching stages, which increases the hardware cost of the interconnects and adversely affects their efficiency and scalability. In this paper, we present a design of an all-optical lightpath concentrator based on WOC and WDM crossbar switches [2] [3]. The proposed design is nonblocking in both domains, highly scalable, cost-efficient, and can switch signals in both space and wavelength domains without the need of separate wavelength conversion stage.

In the future, we want to simulate the proposed design of the all-optical lightpath concentrator to study its performance in real-life scenarios. We also plan to perform a detailed cost analysis of the proposed lightpath concentrator design. An investigation of needs for priority based lightpath concentrators and its design is also an interesting future research direction.

\section{References}

[1] Y. Leung, "Lightpath Concentrators for All-Optical Networks," Journal of Lightw. Technol., Vol. 24, no. 9, pp. 3259-3267, Sept. 2006.

[2] H. S. Hamza and J. S. Deogun, "WDM Optical Interconnects: A Balanced Design Approach,” IEEE/ACM Trans. Networking, vol. 15, no. 6, pp. 1565-1578, Dec. 2007.

[3] H. S. Hamza and J. S. Deogun, “ Wavelength exchanging cross-connect (WEX) - A new class of photonic cross-connect architectures," Journal of Lightw. Technol., vol. 24, no. 3, pp. 1101-1111, Mar. 2006.

[4] Y. Yang and J. Wang, "Designing WDM Optical Interconnects with Full Connectivity by Using Limited Wavelength Conversion," IEEE Trans. Computers, Vol. 53, no. 12, pp. 1547-1556, Dec. 2006.

[5] X. Qin and Y. Yang, "Nonblocking WDM Switching Networks with Full and Limited Wavelength Conversion," IEEE Trans. Comm., Vol. 50, no. 12, pp. 2032 2041, Dec. 2002.

[6] J. Bruce III, C. Zuhlke, D. Alexander, and J. Deogun, "Thermally Related Fluorescence Hysteresis of Semiconductor Quantum Dots," submitted to Nonotechnology, April 2010. 